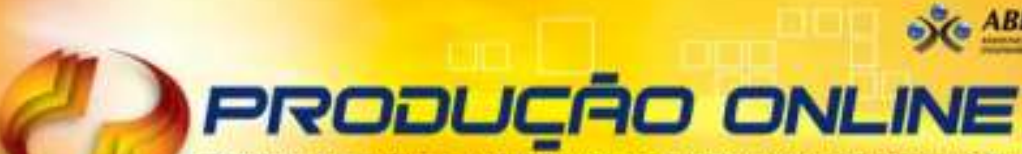 \\ REVISTA CIENTIFICA ELETRÖNICA DE ENGENHARIA DE PRODUÇĀO \\ ISSN 1676-190
}

\section{FATORES ORGANIZACIONAIS INTERNOS QUE FACILITAM OU DIFICULTAM A INOVAÇÃO DE PRODUTOS: ESTUDO DE CASO EM UMA EMPRESA QUE UTILIZA A TIPOLOGIA ETO}

\section{INTERNAL ORGANIZATIONAL FACTORS WHICH FACILITATE OR INHIBIT PRODUCT INNOVATION: A CASE STUDY IN A COMPANY USING ETO PRODUCTION STRATEGY}

\author{
Rogério Gonçalves Donha*E-mail: rogerio.donha@gmail.com \\ Márcia Regina Neves Guimarães* E-mail: mrng@ufscar.br \\ *Universidade Federal de São Carlos (UFSCar), Sorocaba, SP, Brasil
}

Resumo: A inovação é considerada importante quando se trata da competitividade das organizações em geral. Assim, a compreensão dos fatores que facilitam e também dos que dificultam a sua prática torna-se relevante. Nesse contexto, esse artigo tem por objetivo identificar fatores organizacionais internos que podem facilitar ou inibir a inovação de produto no desenvolvimento de produtos ETO (engenharia sob encomenda ou engineer to order). Trata-se de uma pesquisa qualitativa que utiliza o estudo de caso único em uma empresa multinacional como método de procedimento. Entre os principais resultados é possível citar, como fatores internos à organização que favorecem a inovação, a liberdade e a autonomia na condução de projetos, a disponibilidade de recursos financeiros, o aprendizado contínuo e o compartilhamento de experiências e a existência de indivíduos chave. Por outro lado, como inibidores, destacam-se: a falta de tempo por alta carga de trabalho, a política de treinamentos ineficiente e a falta de integração interfuncional.

Palavras-chave: Fatores Organizacionais. Desenvolvimento de produtos. Engenharia sob encomenda. Inovação.

\begin{abstract}
Innovation is considered important when it comes to the competitiveness of organizations in general. Thus, the understanding of the factors that facilitate and also those that hinder their practice becomes relevant. In this context, this article aims to identify internal organizational factors that may facilitate or inhibit product innovation in the development of ETO (engineer to order) products. This qualitative research uses the single case study in a multinational company as a procedure method. Among the main results, internal organizational factors that favor innovation are cited: freedom and autonomy in the conduct of projects, availability of financial resources, continuous learning and the sharing of experiences and the existence of key individuals. On the other hand, as inhibitors, the following stand out: lack of time due to high workload, inefficient training policy and lack of crossfunctional integration.
\end{abstract}

Keywords: Organizational Factors. Product development. Engineering to order. Innovation.

\section{INTRODUÇÃO}

As organizações que só iniciam o processamento de uma ordem interna através do pedido de um cliente são denominadas empresas orientadas por pedidos 
e têm no seu caso mais extremo as que adotam a tipologia ETO (HOOSHMAND; KÖHLER; KORFF-KRUMM, 2016).

Apesar de ainda não ter uma fronteira bem definida - pois é fato que ainda não existe um consenso geral na literatura quanto a correta definição de ETO (WILLNER et al., 2016) - a ETO (engineering to order ou engenharia sob encomenda) é conceituada por grande parte dos autores como o sistema de produção que possui o seu ponto de desacoplamento da cadeia de suprimentos na fase de concepção (GOSLING; NAIM, 2009). Dentre as inúmeras características típicas da ETO estão a flexibilidade, o alto lead time, a mistura de componentes customizados com componentes padrões, as mudanças frequentes, os riscos de prazo e as incertezas quanto à data de entrega.

Já o modelo do processo de desenvolvimento de produtos da ETO, que é o responsável por criar novos produtos mais competitivos e em menos tempo, possui algumas características específicas como afirmam Rozenfeld et al. (2006). Os autores chamam atenção no modelo do PDP ETO para a simplificação do planejamento estratégico, a sofisticação do planejamento do projeto (que deve conter a parte das atividades de projeto informacional e de lançamento), o fato da fase de detalhamento poder ocorrer em mais de um ciclo, a simplificação da fase de preparação da produção (já que não haverá lote piloto) e a inexistência de uma fase de lançamento do produto (que é substituída por uma fase de homologação que visa certificar o atingimento das metas previstas para o produto).

Porém, de forma semelhante, a inovação também é tida como essencial para a competitividade das empresas e está diretamente relacionada com a criação de novos produtos. A definição da inovação pode ser tida como a transformação de ideias em novidades com valor agregado, quando se cria um novo ou melhorado produto, serviço ou processo para se diferenciar no mercado (OECD, 2005; BAREGHEH; ROWLEY; SAMBROOK, 2009; CROSSAN; APAYDIN, 2010). Dentre os tipos de inovação, talvez a mais comum seja a inovação tecnológica (que atua em mudanças de produtos e processos). Este tipo de inovação tem no desenvolvimento de novos produtos uma maneira de se manifestar (PISTORIUS; UTTERBACK, 1997).

É importante ressaltar que o sucesso da inovação depende da organização combinar uma série de capacidades e identificar as barreiras que podem resultar em 
falhas na introdução de novos produtos e/ou processos (D’ESTE et al. 2012). Assim, diversos fatores organizacionais podem influenciar o sucesso ou o fracasso da inovação de produtos e processos que ocorrem durante o desenvolvimento de um produto.

Tais fatores podem ter origem interna ou externa à organização e podem se comportar de várias maneiras devido a sua natureza dinâmica. Portanto, conhecer o processo de desenvolvimento de produtos da organização é vital para poder integrar os componentes que trabalham em conjunto para criar e reforçar o tipo de ambiente que permite que a inovação tecnológica floresça (TIDD; BESSANT; PAVITT, 2008).

Diante do panorama apresentado, o objetivo deste trabalho é o de identificar os fatores organizacionais internos que facilitam e também os que dificultam a inovação de produto em uma empresa que desenvolve produtos utilizando-se da tipologia de produção ETO. Atingir este propósito torna a pesquisa importante pelo fato de ser crucial que os administradores entendam os processos organizacionais e que desenvolvam a habilidade de identificar e usar diferentes abordagens à administração e a organização como afirma Morgan (2002).

Para cumprir o que foi proposto, este trabalho de gênero empírico adotou uma abordagem exploratória e o estudo de caso único em uma empresa que desenvolve sistemas de acionamentos sob encomenda para os mais diversos ramos da indústria como método de procedimento.

A partir desta introdução, na seção 2 deste artigo é apresentada uma revisão da literatura sobre inovação e sobre como os fatores organizacionais a influenciam. Posteriormente, o método de pesquisa e os resultados da coleta de dados formam as seções 3 e 4 respectivamente. Por fim, a seção 5 faz as considerações finais.

\section{REVISÃO BIBLIOGRÁFICA}

O mercado tem sofrido constantes mudanças ao longo do tempo como, por exemplo, alternâncias no campo socioeconômico, nas legislações ou no lançamento de novos produtos por parte da concorrência (TIDD; BESSSANT; PAVITT, 2008). Por isso as empresas precisam estar prontas para aproveitar estas mudanças e as oportunidades que a tecnologia oferece para reagir e responder as demandas e estilos de vida dos clientes (BAREGHEH; ROWLEY; SAMBROOK, 2009). Uma 
característica importante das empresas, que visa garantir sua competitividade frente à forte concorrência que surge, é a sua capacidade de inovação. Competir com o tempo reflete uma crescente pressão sobre as empresas. A inserção de novos produtos no mercado, fazer algo que ninguém mais pode ou possuir características diferenciadas em produtos mais maduros como qualidade, modelo ou customização, promovem uma melhora no desempenho mercadológico da empresa (TIDD; BESSSANT; PAVITT, 2008).

Por conta disso é amplamente reconhecida a crescente importância da inovação para a competitividade. Esse reconhecimento ocorre de forma intensa tanto no meio acadêmico quanto no meio empresarial (STEFANOVITZ; NAGANO, 2014). Na literatura é possível perceber que vários autores (CARAYANNIS; GONZALEZ, 2003; BECHEIKH; LANDRY; AMARA, 2006; DAMANPOUR; WISCHNEVSKY, 2006; HAUSER; TELLIS; GRIFFIN, 2006; TIDD; BESSSANT; PAVITT, 2008; MADRID-GUIJARRO; GARCIA; VAN AUKEN, 2009; GUNDAY et al.,2011, BRUNO-FARIA; FONSECA, 2014; SANTOS et al., 2015; MARZALL; SANTOS; GODOY, 2016) ressaltam a importância da inovação para as organizações se manterem competitivas no contexto atual.

Para a OECD (2005), a inovação se refere à implementação de um produto ou de um processo novo ou significativamente melhorado, de um novo método de marketing ou de um novo método organizacional nas atividades comerciais, na organização do trabalho ou nas relações externas. Para Crossan e Apaydin (2010), a inovação ocorre quando se produz ou se adota uma novidade de valor com valor agregado, quando se renova ou se amplia produtos, serviços ou mercados, quando se desenvolve novos métodos de produção ou quando se estabelece novos sistemas de gerenciamento. Ainda conforme a OECD (2005), existem quatro tipos de inovação: inovação de produto, inovação de processo, inovação de marketing e inovação organizacional. As inovações de produto e de processo são chamadas de inovações tecnológicas.

A inovação de produto, foco do presente estudo, pode ser definida como a introdução de um bem ou serviço que seja novo ou que tenha sofrido aperfeiçoamento significativo no que diz respeito às suas características ou modo de utilização. Essas modificações incluem melhoramentos importantes nas 
especificações técnicas, nos componentes e materiais, no software incorporado, na facilidade de uso ou nas características funcionais (HAGE; MEEUS, 2009).

No ambiente organizacional, diversos fatores podem influenciar o sucesso ou o fracasso em um processo de inovação; e o fracasso não é algo incomum. A inovação é por natureza um negócio de risco e é importante lembrar que ela lida com muitas incertezas (TIDD; BESSANT; PAVITT, 2008; MADRID-GUIJARRO; GARCIA; VAN AUKEN, 2009; DEMIRBAS, HUSSAIN, MATLAY, 2011). O sucesso da inovação depende da organização combinar uma série de capacidades e identificar as barreiras que podem resultar em falhas na introdução de novos produtos e/ou processos (D’ESTE et al. 2012).

Tidd, Bessant e Pavitt (2008) ressaltam que a "organização inovadora" é um conjunto integrado de componentes que trabalham em conjunto para criar e reforçar o tipo de ambiente que permite que a inovação floresça. Os autores afirmam que, embora os estudos de organizações inovadoras possam ser criticados por tomar uma visão muito estreita, é possível extrair a partir destes estudos um conjunto de componentes que parecem estar ligados ao sucesso.

Por sua vez, Carayannis e Gonzalez (2003) destacam que os fatores condicionantes à inovação podem ser de origem interna ou externa à organização. Os fatores externos à organização são aqueles que vêm do ambiente e os fatores internos são relativos à organização em si. Outros autores também salientam que os fatores organizacionais que afetam a inovação podem estar relacionados à aspectos internos ou externos à organização (HADJIMANOLIS, 2003; BECHEICK; LANDRY; AMARA, 2006; MADRID-GUIJARRO; GARCIA; VAN AUKEN, 2009; DEMIRBAS; HUSSAIN; MATLAY, 2011).

Para Machado e Vasconcellos (2007), muitos trabalhos mostram que características da cultura organizacional estão diretamente relacionadas à existência de práticas inovadoras. Valencia, Valle e Jiménez (2010), em estudo realizado em empresas do sudeste da Espanha, concluíram que a cultura organizacional, dependendo dos fatores que fomenta, pode tanto estimular quanto inibir a inovação de produto. Para os autores, a cultura organizacional é um elemento fundamental quando se trata desse tipo de inovação. Os resultados da pesquisa mostraram que empresas com culturas adhocráticas, ou seja, culturas que fomentam a criatividade, o empreendedorismo e a assunção a riscos, possuem maior relação com a geração 
de inovação de produtos. Por outro lado, empresas com culturas hierarquizadas com ênfase no controle interno, na fiel aderência a regras e regulamentos e na orientação interna, apresentam menor relação com a inovação de produto (VALENCIA; VALLE; JIMÉNEZ, 2010). Outros autores (JANTZ, 2014; WANG, ZHAO; THORNHILL, 2015) reforçam essa relação entre a cultura organizacional e a prática da inovação.

Lin e McDonough III (2011) acrescentam que o líder deve estimular a criatividade dos trabalhadores por meio do incentivo à exploração de ideias, ao tratamento de problemas por diferentes ângulos e ao constante questionamento no que se refere às novas maneiras de se fazer o que vem sendo feito. Dessa forma, segundo os autores, os líderes conseguem aumentar a interação e a colaboração entre os trabalhadores através da criação de normas culturais. Eles devem se tornar "modelos" e promover comportamentos que estimulem a inovação.

Outros autores (LAM, 2004; BECHEIKH; LANDRY; AMARA, 2006) citam fatores internos à organização que estão relacionados com a prática da inovação. Enquanto Lam (2004) afirma que a estrutura de uma organização pode tanto facilitar quanto inibir a inovação, Becheikh, Landry e Amara (2006), a partir de uma revisão da literatura que compreendeu o período de 1993 a 2003, concluem que existe, além de fatores externos, um conjunto de fatores internos que possuem uma relação fortemente significativa com o comportamento inovador de uma organização. 0 Quadro 1 foi extraído deste estudo.

Quadro 1 - Determinantes internos para a inovação (continua)

\begin{tabular}{|c|c|c|}
\hline CATEGORIA & SUBCATEGORIA & VARIÁVEIS \\
\hline \multirow{4}{*}{$\begin{array}{l}\text { Características } \\
\text { gerais da } \\
\text { organização }\end{array}$} & \multirow{4}{*}{ - } & Tamanho da empresa \\
\hline & & Idade da empresa \\
\hline & & Estrutura de propriedade nacional ou estrangeira \\
\hline & & Desempenho passado da empresa \\
\hline \multirow{7}{*}{$\begin{array}{l}\text { Estratégia } \\
\text { Competitiva }\end{array}$} & Definição da estratégia & Existência de uma estratégia de orientação definida \\
\hline & \multirow{3}{*}{ Estratégia corporativa } & Estratégia de diversificação \\
\hline & & Exportação/Internacionalização \\
\hline & & Crescimento externo $\mathrm{x}$ crescimento interno \\
\hline & \multirow{3}{*}{ Estratégia de negocio } & Estratégia de diferenciação \\
\hline & & Estratégia de redução de custos \\
\hline & & Mecanismos de proteção \\
\hline
\end{tabular}


Quadro 1 - Determinantes internos para a inovação (conclusão)

\begin{tabular}{|c|c|c|}
\hline CATEGORIA & SUBCATEGORIA & VARIÁVEIS \\
\hline \multirow{5}{*}{$\begin{array}{l}\text { Estrutura da } \\
\text { empresa }\end{array}$} & \multirow{2}{*}{ Formalização } & Estrutura formal \\
\hline & & Estrutura flexível \\
\hline & \multirow{2}{*}{ Centralização } & Centralização da tomada de decisões \\
\hline & & Empoderamento dos funcionários \\
\hline & Interação & Interação entre as unidades da empresa \\
\hline Controle & - & Controle financeiro $\mathrm{x}$ controle estratégico \\
\hline \multirow[t]{3}{*}{$\begin{array}{l}\text { Cultura } \\
\text { organizacional }\end{array}$} & - & Resistência às mudanças \\
\hline & & Gestão da qualidade total/melhoria contínua \\
\hline & & Cultura de suporte à inovação \\
\hline \multirow{6}{*}{$\begin{array}{l}\text { Time de } \\
\text { gerenciamento }\end{array}$} & \multirow{3}{*}{ Variáveis de liderança } & Presença de um líder de projetos \\
\hline & & Características do diretor executivo \\
\hline & & Substituição do diretor executivo \\
\hline & \multirow{3}{*}{$\begin{array}{l}\text { Variáveis ligadas a } \\
\text { gerência }\end{array}$} & Qualificação e experiência \\
\hline & & Percepção de riscos e custos relacionados à inovação \\
\hline & & Percepção do retorno à inovação \\
\hline \multirow{10}{*}{ Áreas funcionais } & P\&D & Estratégias de P\&D \\
\hline & \multirow{2}{*}{ Recursos humanos } & Qualificação e experiência dos trabalhadores \\
\hline & & Estratégias de recursos humanos \\
\hline & \multirow{2}{*}{ Operações e produção } & Equipamentos e tecnologias avançadas \\
\hline & & Grau de utilização da capacidade de produção \\
\hline & \multirow{2}{*}{ Marketing } & Estratégias de marketing \\
\hline & & Monitoramento de concorrentes \\
\hline & \multirow{3}{*}{ Financeiro } & Autonomia financeira \\
\hline & & Volume de negócios/lucro \\
\hline & & Disponibilidade financeira \\
\hline
\end{tabular}

Fonte: Adaptado de BECHEIKH; LANDRY; AMARA (2006)

Além de levantar os fatores organizacionais internos que afetam a inovação de alguma maneira, a revisão de Becheikh, Landry e Amara (2006) também aponta para o fato de que um fator pode ser classificado como catalisador a inovação em uma pesquisa e em outra como inibidor. O fator "estrutura formal" que está relacionado à estrutura organizacional, por exemplo, é citado como um facilitador a inovação em dois artigos, como uma barreira por outro artigo e como não significante em outros dois estudos (BECHEIKH; LANDRY; AMARA, 2006).

Apesar de ser uma das referências mais importantes no estudo de fatores organizacionais que afetam a inovação, esta não é a única revisão já feita sobre o 
assunto. O pesquisador Chryssochoidis (2003) analisou aproximadamente 400 jornais científicos durante os anos de 1991 a 2001 e Valladares, Vasconcellos e Di Serio (2014) aplicaram uma metodologia sistemática de 1991 a 2011 . O Quadro 2 foi elaborado baseado nestas três revisões e em outras obras devidamente referenciadas na última coluna. Os fatores foram organizados de uma maneira que ressaltasse a dicotomia entre alguns fatores.

Quadro 2 - Fatores internos à organização que afetam a inovação

(continua)

\begin{tabular}{|c|c|}
\hline FATORES ORGANIZACIONAIS & REFERÊNCIAS \\
\hline $\begin{array}{l}\text { Tamanho da empresa (empresas grandes } \\
\text { apoiam as atividades de risco e as } \\
\text { pequenas são mais eficientes na criação). }\end{array}$ & $\begin{array}{l}\text { Becheikh, Landry e Amara (2006); Chryssochoidis } \\
\text { (2003). }\end{array}$ \\
\hline $\begin{array}{l}\text { Idade da empresa (empresas antigas } \\
\text { acumulam experiência e conhecimento e, } \\
\text { por outro lado, estabelecem procedimentos } \\
\text { e rotinas prejudiciais). }\end{array}$ & $\begin{array}{l}\text { Becheikh, Landry e Amara (2006); Chryssochoidis } \\
\text { (2003). }\end{array}$ \\
\hline $\begin{array}{l}\text { Desempenho passado da empresa (um bom } \\
\text { desempenho da empresa tende a encorajar } \\
\text { a inovação). }\end{array}$ & Becheikh, Landry e Amara (2006). \\
\hline Estratégia de diversificação. & Becheikh, Landry e Amara (2006). \\
\hline $\begin{array}{l}\text { Existência de exportações } \\
\text { (internacionalização). }\end{array}$ & Becheikh, Landry e Amara (2006). \\
\hline $\begin{array}{l}\text { Estratégia de diferenciação ou temor por } \\
\text { canibalizar a venda de produtos já } \\
\text { estabelecidos. }\end{array}$ & $\begin{array}{l}\text { Becheikh, Landry e Amara (2006); Hadjimanolis } \\
\text { (2003). }\end{array}$ \\
\hline $\begin{array}{l}\text { Estratégia de redução de custos (copiar } \\
\text { produtos ao invés de gastar com P\&D). }\end{array}$ & Becheikh, Landry e Amara (2006). \\
\hline $\begin{array}{l}\text { Mecanismos de proteção (registro de } \\
\text { patentes, tecnologia, proteção de segredos } \\
\text { industriais, etc.). }\end{array}$ & Becheikh, Landry e Amara (2006). \\
\hline $\begin{array}{l}\text { Ausência de } \text { gestão por resultados ou } \\
\text { sistema de recompensas para inovaçãa com } \\
\text { incentivos para pesquisa } r \\
\text { desenvolvimento. }\end{array}$ & $\begin{array}{l}\text { Becheikh, Landry e Amara (2006); Carayannis e } \\
\text { Gonzalez (2003); Chryssochoidis (2003); } \\
\text { Hadjimanolis (2003); Valladares, Vasconcellos e Di } \\
\text { Serio (2014); Melo e Bagno (2017). }\end{array}$ \\
\hline $\begin{array}{l}\text { Planejamento estratégico ineficiente ou a } \\
\text { existência de uma estratégia de orientação } \\
\text { definida que leve a um planejamento claro. }\end{array}$ & $\begin{array}{l}\text { Becheikh, Landry e Amara (2006); Carayannis e } \\
\text { Gonzalez (2003); Chryssochoidis (2003); } \\
\text { Hadjimanolis (2003). }\end{array}$ \\
\hline $\begin{array}{l}\text { Conjunto adequado de pessoas } \\
\text { (conhecimento diversificado, qualificação, } \\
\text { experiência e criatividade). }\end{array}$ & $\begin{array}{l}\text { Becheikh, Landry e Amara (2006); Carayannis e } \\
\text { Gonzalez (2003); Tidd, Bessant e Pavitt (2008); } \\
\text { Valladares, Vasconcellos e Di Serio (2014). }\end{array}$ \\
\hline Espírito de equipe. & Carayannis e Gonzalez (2003). \\
\hline
\end{tabular}




\begin{tabular}{|c|c|}
\hline FATORES ORGANIZACIONAIS & REFERÊNCIAS \\
\hline $\begin{array}{l}\text { Cultura e motivação para a inovação ou } \\
\text { resistência em contexto cultural. }\end{array}$ & $\begin{array}{l}\text { Becheikh, Landry e Amara (2006); Carayannis e } \\
\text { Gonzalez (2003), Chryssochoidis (2003); } \\
\text { Hadjimanolis (2003); Morgan (2002); Tidd, Bessant e } \\
\text { Pavitt (2008). }\end{array}$ \\
\hline $\begin{array}{l}\text { Senso de acomodação (tendência a manter } \\
\text { tudo como está e evitar mudanças). }\end{array}$ & $\begin{array}{l}\text { Carayannis e Gonzalez (2003); Chryssochoidis } \\
\text { (2003); Hadjimanolis (2003). }\end{array}$ \\
\hline $\begin{array}{l}\text { Aprendizado contínuo e compartilhamento } \\
\text { de experiências. }\end{array}$ & $\begin{array}{l}\text { Carayannis e Gonzalez (2003); Chryssochoidis } \\
\text { (2003); Tidd, Bessant e Pavitt (2008); Valladares, } \\
\text { Vasconcellos e Di Serio (2014); Almeida et al. (2016). }\end{array}$ \\
\hline $\begin{array}{l}\text { Falta de tempo por alta carga de trabalho ou } \\
\text { tempo suficiente para se dedicar a inovação. }\end{array}$ & $\begin{array}{l}\text { Becheikh, Landry e Amara (2006); Chryssochoidis } \\
\text { (2003); Hadjimanolis (2003). }\end{array}$ \\
\hline $\begin{array}{l}\text { Existência de projetos de longo prazo ou } \\
\text { priorização de resultados de curto prazo. }\end{array}$ & $\begin{array}{l}\text { Becheikh, Landry e Amara (2006); Carayannis e } \\
\text { Gonzalez (2003); Chryssochoidis (2003); } \\
\text { Hadjimanolis (2003). }\end{array}$ \\
\hline Controle financeiro. & Becheikh, Landry e Amara (2006). \\
\hline $\begin{array}{l}\text { Falta de recursos financeiros ou a sua } \\
\text { disponibilidade. }\end{array}$ & $\begin{array}{l}\text { Becheikh, Landry e Amara (2006); Carayannis e } \\
\text { Gonzalez (2003); Chryssochoidis (2003); } \\
\text { Hadjimanolis (2003); Valladares, Vasconcellos e Di } \\
\text { Serio (2014). }\end{array}$ \\
\hline $\begin{array}{l}\text { Existência de indivíduos chave ou a sua } \\
\text { falta. }\end{array}$ & $\begin{array}{l}\text { Chryssochoidis (2003); Hadjimanolis (2003); Morgan } \\
\text { (2002); Tidd, Bessant e Pavitt (2008). }\end{array}$ \\
\hline $\begin{array}{l}\text { Existência de uma política de treinamento } \\
\text { dos recursos humanos ou a sua ausência. }\end{array}$ & $\begin{array}{l}\text { Becheikh, Landry e Amara (2006); Hadjimanolis } \\
\text { (2003); Tidd, Bessant e Pavitt (2008); Almeida et al. } \\
(2016) .\end{array}$ \\
\hline $\begin{array}{l}\text { Utilização de equipamentos e tecnologias } \\
\text { avançadas. }\end{array}$ & Becheikh, Landry e Amara (2006). \\
\hline $\begin{array}{l}\text { Utilização de softwares para gestão da } \\
\text { inovação e do conhecimento. }\end{array}$ & Chryssochoidis (2003). \\
\hline $\begin{array}{l}\text { Rigidez de estruturas hierárquicas ou a } \\
\text { promoção de grupos interfuncionais } \\
\text { orientado a projetos. }\end{array}$ & $\begin{array}{l}\text { Becheikh, Landry e Amara (2006); Carayannis e } \\
\text { Gonzalez (2003); Chryssochoidis (2003); } \\
\text { Hadjimanolis (2003); Morgan (2002); Valladares, } \\
\text { Vasconcellos e Di Serio (2014). }\end{array}$ \\
\hline $\begin{array}{l}\text { Jogos políticos internos e problema de } \\
\text { obstrução por parte } \\
\text { departamentos. }\end{array}$ & Chryssochoidis (2003); Hadjimanolis (2003). \\
\hline Integração interfuncional ou a sua falta. & $\begin{array}{l}\text { Becheikh, Landry e Amara (2006); Chryssochoidis } \\
\text { (2003); Hadjimanolis (2003); Valladares, Vasconcellos } \\
\text { e Di Serio (2014). }\end{array}$ \\
\hline $\begin{array}{l}\text { Centralização da tomada de decisões pelos } \\
\text { líderes ou empoderamento dos funcionários. }\end{array}$ & $\begin{array}{l}\text { Becheikh, Landry e Amara (2006); Carayannis e } \\
\text { Gonzalez (2003); Chryssochoidis (2003); } \\
\text { Hadjimanolis (2003); Valladares, Vasconcellos e Di } \\
\text { Serio (2014). }\end{array}$ \\
\hline $\begin{array}{l}\text { Problemas na comunicação entre } \\
\text { departamentos ou fluxo de informações } \\
\text { eficiente. }\end{array}$ & $\begin{array}{l}\text { Chryssochoidis (2003); Hadjimanolis (2003); Tidd, } \\
\text { Bessant e Pavitt (2008); Valladares, Vasconcellos e } \\
\text { Di Serio (2014). }\end{array}$ \\
\hline Estratégias de P\&D. & Becheikh, Landry e Amara (2006). \\
\hline $\begin{array}{l}\text { Estratégias de marketing (acompanhamento } \\
\text { das necessidades dos clientes). }\end{array}$ & Becheikh, Landry e Amara (2006). \\
\hline Monitoramento de concorrentes. & Becheikh, Landry e Amara (2006). \\
\hline
\end{tabular}


Quadro 2 - Fatores internos à organização que afetam a inovação

(conclusão)

\begin{tabular}{|l|l|}
\hline \multicolumn{1}{|c|}{ FATORES ORGANIZACIONAIS } & \multicolumn{1}{c|}{ REFERÊNCIAS } \\
\hline $\begin{array}{l}\text { Presença de um líder de projetos ou a sua } \\
\text { ausência. }\end{array}$ & $\begin{array}{l}\text { Becheikh, Landry e Amara (2006); Hadjimanolis } \\
\text { (2003). }\end{array}$ \\
\hline $\begin{array}{l}\text { Características pessoais do diretor } \\
\text { executivo. }\end{array}$ & Becheikh, Landry e Amara (2006). \\
\hline Diretor com liderança transformacional. & Becheikh, Landry e Amara (2006). \\
\hline Qualificação e experiência do(s) gerente(s). & Becheikh, Landry e Amara (2006). \\
\hline $\begin{array}{l}\text { Dirigentes com habilidades que identificam, } \\
\text { apoiam, incentivam e assumem riscos em } \\
\text { prol da inovação ou a falta de apoio e } \\
\text { aversão exacerbada ao risco. }\end{array}$ & $\begin{array}{l}\text { Becheikh, Landry e Amara (2006); Carayannis e } \\
\text { Gonzalez (2003); Chryssochoidis (2003); } \\
\text { Hadjimanolis (2003); Tidd, Bessant e Pavitt (2008); } \\
\text { Valladares, Vasconcellos e Di Serio (2014); Almeida } \\
\text { et al. (2016). }\end{array}$ \\
\hline Falta de tolerância à falha. & Hadjimanolis (2003). \\
\hline
\end{tabular}

Concluindo, existem inúmeros fatores organizacionais apontados pela literatura como determinantes à inovação. Assim, a seção 3 apresenta uma metodologia de pesquisa que busca encontrar quais destes fatores estão presentes no processo de desenvolvimento de produtos sob encomenda (PDP ETO) e, então, relacioná-los com a capacidade de inovação de produtos.

\section{METODOLOGIA DE PESQUISA}

Este artigo busca tornar explícitos os fatores organizacionais que afetam a inovação de produtos no PDP ETO e, como distinguiu Demo (1995), a pesquisa cujo gênero se dedica a codificar uma face mensurável da realidade social é a pesquisa empírica. Flynn et al. (1990) definem empírico como o conhecimento baseado na vida real através de observações e propõe uma abordagem sistemática em sua pesquisa através de um roteiro de seis passos, que vai desde a escolha da fundamentação teórica até a publicação do trabalho.

Em consonância com este roteiro, ao levar em conta os objetivos e a abordagem, esta pesquisa se classifica como exploratória e qualitativa. Gil (2002) corrobora com esta escolha ao caracterizar a pesquisa exploratória como a que tem como objetivo proporcionar maior familiaridade com o problema com vistas a torná-lo mais explícito. Já Mazzotti e Gewandsznajder (1998) caracterizam a pesquisa qualitativa como aquela que busca o detalhamento de um ambiente.

Sodhi e Tang (2014) ressaltam a importância de uma pesquisa se posicionar adequadamente no fluxo atual de pesquisa, Assim, visando justificar o caráter 
exploratório desta pesquisa, foram realizadas buscas em importantes bancos de dados, mas os resultados em nada puderam acrescentar para responder ao problema de pesquisa. Portanto se justifica a abordagem exploratória desta pesquisa.

Quanto ao método de pesquisa, o estudo de caso único se mostrou o mais apropriado, pois como afirma Yin (2010), este é o método preferido quando se busca examinar eventos contemporâneos sem poder manipular os comportamentos relevantes. Já a coleta de dados se valeu de observação participante (entre 2015 e 2016) e entrevistas semiestruturadas nos fatores do Quadro 2 (ocorridas nos primeiros meses de 2015). O trabalho de Yin (2010) destaca que as múltiplas fontes de evidência nos estudos de caso permitem abordar uma variação maior de aspectos históricos e comportamentais. Sua principal vantagem é um processo de corroboração e triangulação que proporciona várias avaliações do mesmo fenômeno e, consequentemente, diminui os problemas potenciais de validade da pesquisa (YIN, 2010). O Quadro 3 apresenta a classificação da pesquisa vista até aqui de forma resumida.

Quadro 3 - Classificação da pesquisa

\begin{tabular}{|l|l|l|l|l|}
\hline $\begin{array}{c}\text { Classificação } \\
\text { quanto ao } \\
\text { gênero }\end{array}$ & $\begin{array}{c}\text { Classificação } \\
\text { quanto à } \\
\text { abordagem }\end{array}$ & $\begin{array}{c}\text { Classificação } \\
\text { quanto aos } \\
\text { objetivos }\end{array}$ & $\begin{array}{c}\text { Classificação } \\
\text { quanto ao } \\
\text { método de } \\
\text { pesquisa }\end{array}$ & $\begin{array}{c}\text { Classificação quanto ao } \\
\text { método de coleta de } \\
\text { dados }\end{array}$ \\
\hline Empírica & Exploratória & Qualitativa & $\begin{array}{l}\text { Estudo de caso } \\
\text { único }\end{array}$ & $\begin{array}{l}\text { • Entrevistas } \\
\text { • Observação participante }\end{array}$ \\
\hline
\end{tabular}

Quanto à confiabilidade das entrevistas, a medida do alfa de Cronbach proposta por Hair et al. (2009) e equacionado por Leontitsis e Page (2007) foi calculada através da tabulação das respostas em uma escala de Likert de 1 a 3 sendo: (1) fator inibidor à inovação de produtos, (2) fator neutro para a inovação de produtos e (3) fator catalisador à inovação de produtos. O resultado foi um alfa de Cronbach de 0,526 como pode ser visto na Figura 1. 
Figura 1 - Índice de confiabilidade

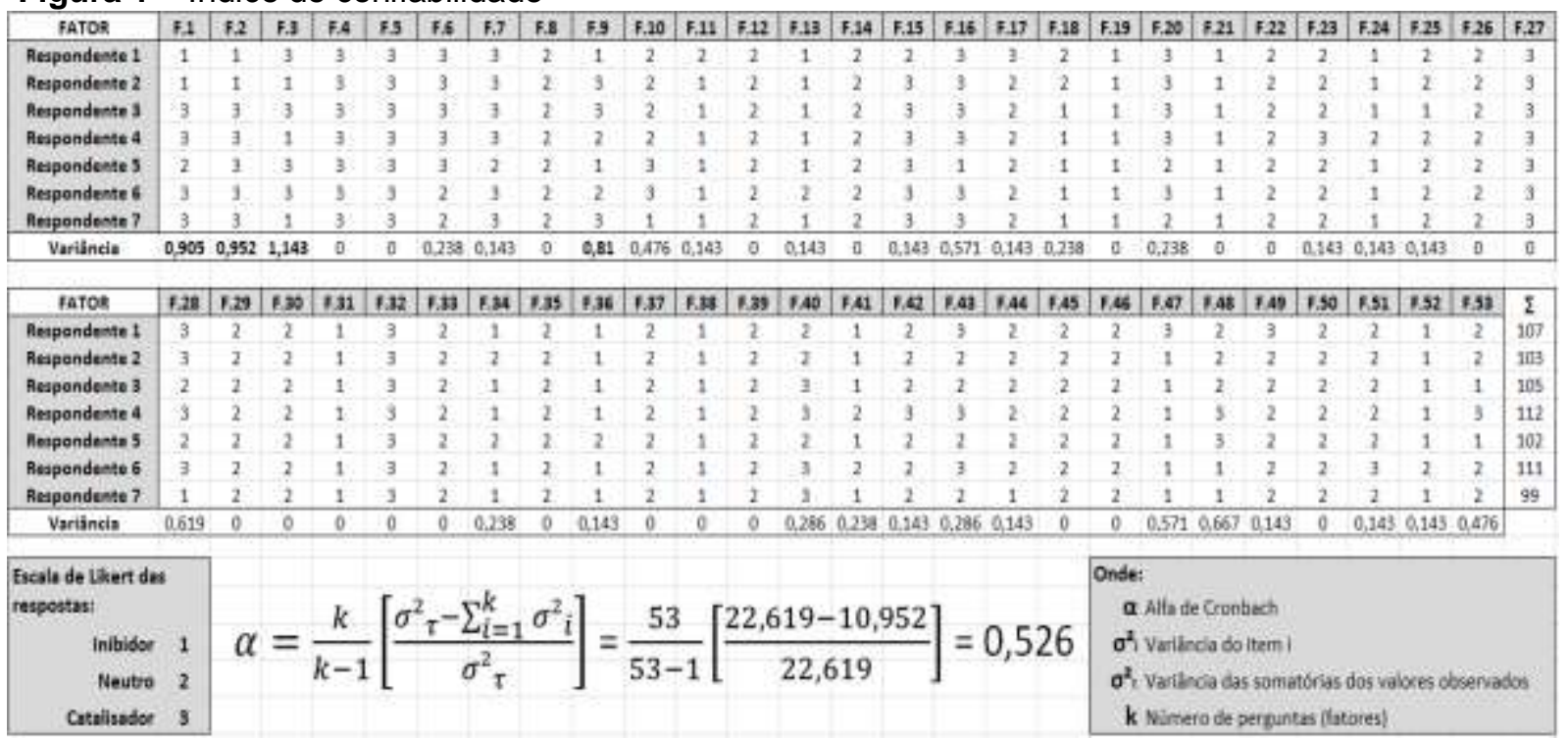

O limite inferior para o alfa de Cronbach geralmente aceito é 0,70, mas esse valor pode ser diminuído para 0,60 em pesquisas exploratórias (HAIR et al., 2009). Para resolver este problema, os quatro fatores que mais divergiram entre os respondentes foram excluídos sendo: tamanho da organização, idade da organização, estrutura de propriedade estrangeira da organização e estratégias para redução de custos. Assim, os resultados da pesquisa passaram a ser considerados confiáveis com um alfa de Cronbach de 0,74 como pode ser visto na Figura 2.

Figura 2 - Índice de confiabilidade tratado

\begin{tabular}{|c|c|c|c|c|c|c|c|c|c|c|c|c|c|c|c|c|c|c|c|c|c|c|c|c|c|c|c|}
\hline FATOR & $F 1$ & $\$ 2$ & $\mathrm{~F} \cdot 3$ & F.4 & F.S & $F .6$ & E9 & 7.8 & F.9. & F.10 & F.11 & $F 12$ & $F .13$ & $F .14$ & F.15 & F.16 & E.17 & F.18 & $F .19$ & 7.20 & $F .21$ & 5.22 & F.23 & 8.24 & E.25 & F.26 & F.27 \\
\hline Respondente 1 & & & & 3 & 3 & 3 & 3 & 2 & & 2 & 2 & 2 & 1 & 2 & 2 & 3 & 3 & 2 & 1 & 3 & 1 & 2 & 2 & 1 & 2 & 2 & 3 \\
\hline Respondente 2 & & & & 3 & 3 & 3 & 3 & 2 & & 2 & 1 & 2 & 1 & 2 & 3 & 3 & 2 & 2 & 1 & 3 & 1 & 2 & 2 & 1 & 2 & 2 & 3 \\
\hline Aespondente 3. & & & & 3 & 3 & 3 & 3 & 2 & & 2 & 1 & 2 & 1 & 2 & 3 & 3 & 2 & 1 & 1 & 3 & 1 & 2 & 2 & 1 & 1 & 2 & 3 \\
\hline Respondente 4 & & & & 3 & 3 & 3 & 3 & 2 & & 2 & 1 & 2 & 1 & 2 & 3 & 3 & 2 & 1 & 1 & 9 & 1 & 2 & 3 & 2 & 2 & 2 & 3 \\
\hline Respondente S & & & & 3 & 3 & 3 & 2 & 2 & & 3 & 1 & 2 & 1 & 2 & 3 & $t$ & 2 & 1 & 1 & 2 & 1 & 2 & 2 & 1 & 2 & 2 & 3 \\
\hline Respondente 6 & & & & 3 & 3 & 2 & 3 & 2 & & 3 & 1 & 2 & 2 & 2 & 3 & 3 & 2 & 1 & 1 & 3 & 1 & 2 & 2. & 1 & 2 & 2 & 3 \\
\hline Alespondente? & & & & 3 & 3 & 2 & 3 & 2 & & 1 & 1 & 2 & 1 & 2 & 3 & 3 & 2 & 1 & 1 & 2 & 1 & 2 & 2 & 1 & 2 & 2 & 3 \\
\hline Variintis & & & & 0 & 0 & 0.238 & 0.143 & 0 & $(8 \%)$ & 0.476 & 0.143 & 0 & 0.143 & 0 & 0.123 & 0.571 & 0.143 & 0.238 & 0 & 0.238 & 8 & 0 & 0.193 & 0.143 & 0.143 & 0 & a \\
\hline
\end{tabular}

\begin{tabular}{|c|c|c|c|c|c|c|c|c|c|c|c|c|c|c|c|c|c|c|c|c|c|c|c|c|c|c|c|}
\hline FATCR & 128 & 1.29 & 1.30 & k.ts & 1.32 & 133 & 134 & Has & E.1. & $\mathrm{k} .7$ & 1.:38 & 131 & 140 & $F A 1$ & 502 & 1.43 & 154 & Fas & 1746 & F47 & 1,48 & 5.6 & 180 & 1.51 & 152 & F.33 & 2 \\
\hline Respondente 1 & 3 & 2 & 2 & 1 & 3 & 2 & 1 & 2 & 1 & 2 & 1 & 2 & 2 & 1 & 2 & 3 & 2 & 2 & 2 & 3 & 2 & 3 & 2 & 2 & 1 & 2 & 101 \\
\hline Respendente 2 & 3 & 2 & 2 & 1 & 3 & 2 & 2 & 2 & 1 & 2 & 1 & 2 & 2 & 1 & 2 & 2 & 2 & 2 & 2 & 1 & 2 & 2 & 2 & 2 & 1 & 2 & 97 \\
\hline Respondente 3 & 2 & 2 & 2 & 1 & 3 & 2 & 1 & 2 & 1 & 2 & 1 & 2 & 3 & 1 & 2 & 2 & 2 & 2 & 2 & 1 & 2 & 2 & 2 & 2 & 1 & 1 & 93 \\
\hline Respondente 4 & 3 & 2 & 2 & t & 3 & 2 & 1 & 2 & 1 & 2 & 1 & 2 & 3 & 2 & 3 & $\exists$ & 2 & 2 & 2 & 1 & 3 & 2 & 2 & 2 & 1 & 3 & 203 \\
\hline Respondente 5 & 2 & 2 & 2 & 1 & 3 & 2 & 2 & 2 & 2 & 2 & 1 & 2 & 2 & 1 & 2 & 2 & 2 & 2 & 2 & 1 & 3 & 2 & 2 & 2 & 1 & 1 & 93. \\
\hline Respondente 6 & 3 & 2 & 2 & 1 & 3 & 2 & 1 & 2 & 1 & 2 & 1 & 2 & 3 & 2 & 2 & 3 & 2 & 2 & 2 & 1 & 1 & 2 & 2 & 3 & 2 & 2 & 100 \\
\hline Respondente 7 & 1 & 2 & 2 & 1 & 3 & 2 & 1 & 2 & 1 & 2 & 1 & 2 & 3 & 1 & 2 & 2 & 1 & 2 & 2 & 1 & 1 & 2 & 2 & 2 & 1 & 2 & 39 \\
\hline Varlúneia & 0,619 & a & 0 & 0 & 0 & 0 & 0,238 & 0 & 0,14 & 0 & 0 & 0. & 0,286 & 0,238 & 0,123 & 0,286 & 0,143 & 0 & 0 & 0.571 & 0,667 & 0,143 & 0 & $0,143$. & 0,143 & $0,4 \pi$ & \\
\hline
\end{tabular}
$\begin{array}{lllllllllllllllllllllllllll}\text { Varisncia } & 0,619 & 0 & 0 & 0 & 0 & 0 & 0,238 & 0 & 0,143 & 0 & 0 & 0 & 0,286 & 0,218 & 0,123 & 0,286 & 0,143 & 0 & 0 & 0,571 & 0,667 & 0,143 & 0 & 0,143 & 0,143 & 0,476\end{array}$

$$
\begin{aligned}
& \text { Eicala de Uikent des } \\
& \begin{aligned}
\text { Inibidor } & 1 \\
\text { Neutro } & 2 \\
\text { Gatalisador } & 3
\end{aligned}
\end{aligned} \quad \alpha=\frac{k}{k-1}\left[\frac{\sigma_{\tau}^{2}-\sum_{i=1}^{k} \sigma_{i}^{2} i}{\sigma_{\tau}^{2}}\right]=\frac{49}{49-1}\left[\frac{25,952-7,143}{25,952}\right]=0,74
$$

Onde:

a Afa de Cronbach $\sigma^{2}$ Varsincla do zen:

$\sigma^{2}$, Varänincis des somatoris dos valores observados $k$ Nümé de peguntas (tartres) 
A empresa que serviu como objeto de estudo para esta pesquisa é uma multinacional de origem alemã com mais de 80 anos de mercado e que possui em todo o mundo mais de $14 \mathrm{mil}$ funcionários. Atualmente o faturamento anual ultrapassa os 2,5 bilhões de euros. Seu papel no mercado é o de fornecer soluções em acionamentos para as mais diversas aplicações, resultado de um sistema modular versátil composto de redutores, variadores, moto redutores, conversores de frequência, motores elétricos e servo motores. Além de serviços de consultoria que visam permitir a instalação dos produtos de acordo com a necessidade do cliente. Seus produtos são utilizados em inúmeros segmentos como na indústria química, siderurgia, petrolífera, papel e celulose, alimentação, plásticos, cerâmica, automobilística, etc.

Uma de suas fortes características é a de disponibilizar soluções individuais que vai ao encontro dos requisitos específicos do cliente ou do setor em questão. Para tal, além de ter um bom departamento de engenharia de aplicação, que é responsável por ajudar ao cliente a especificar a melhor solução para o seu acionamento, ela também possui um forte departamento de projetos ETO.

Este departamento é responsável pelo desenvolvimento de projetos que customizem ou modifiquem um equipamento padrão. Ou seja, este departamento é responsável pelas inovações incrementais ou radicais de produtos de acordo com as especificações do cliente que chegam através da engenharia de aplicação ou do departamento de vendas.

\section{APRESENTAÇÃO DOS RESULTADOS}

Os fatores organizacionais foram classificados como catalisadores, neutros/inexistentes ou inibidores à inovação de produtos de acordo com as entrevistas de sete engenheiros/projetistas que atuam diretamente no desenvolvimento de produtos sob encomenda, que no caso da empresa que serviu como objeto de estudo, são sistemas de acionamento. Os resultados são detalhados abaixo:

- Tamanho, idade e estrutura de propriedade estrangeira, assim como pode ser visto na literatura (BECHEICK; LANDRY; AMARA, 2006; CHRYSSOCHOIDIS, 2003), há muita divergência sobre a influência 
destes fatores na inovação. Os resultados desta pesquisa para estes fatores tiveram que ser desconsiderados para garantir a confiabilidade das entrevistas (assim como foi justificado no tópico anterior). O mesmo ocorreu com a "existência de estratégias de redução de custos". Tal fator deixou os entrevistados divididos entre dois grupos: os que enxergam que copiar um produto concorrente trazem inovações para a organização e os que acham que isso em nada acrescenta em matéria de inovações;

- Unânime como catalisadores são: o bom desempenho passado da empresa, a estratégia de diversificação e de diferenciação, a disponibilidade de recursos financeiros e a utilização de equipamentos de alta tecnologia. A observação participante converge para o mesmo resultado e destaca o fato da empresa ter recentemente entrado para o mercado de acionamentos para turbinas eólicas. Através da customização de um produto que já era padrão, a empresa pôde diversificar ainda mais seu mercado de atuação. Essa atitude trouxe inúmeros investimentos e, consequentemente, a oportunidade e a motivação para inovação de produtos. A literatura também corrobora com o resultado ao afirmar que a customização em massa é criadora de vantagem competitiva (diferenciação) e coloca a ETO como a classe mais alta na escala de customização em massa (FOGLIATTO; SILVEIRA; BORENSTEIN, 2012);

- Classificados pela maioria como catalisadores foram: a internacionalização, o conjunto adequado de pessoas, o espírito de equipe, o aprendizado contínuo e o compartilhamento de experiências, a existência de indivíduos chave e o empoderamento dos funcionários. De acordo com a revisão da literatura, a internacionalização tende a exigir mais inovações como apontam Becheikh, Landry e Amara (2006). Já a ETO se caracteriza como uma manufatura de protótipos onde a engenharia se dá por um método empírico onde se cria uma dependência humana de difícil gerenciamento (WALTER; RIES, 1996). Consequentemente, a equipe deve ser adequada, ter espírito de equipe, compartilhar experiências e possuir indivíduos chave. Do contrário, os erros de engenharia seriam constantes. Também ficou claro na observação participante que, devido à complexidade dos projetos ETO 
(GOSLIN; NAIM, 2009) e ao ambiente multiprojetos (DANILOVIC; BÖRJESSON, 2001) de difícil gerenciamento, a liderança prefere deixar a tomada de decisões dos por conta dos projetistas;

- Unânime como neutros/inexistentes têm-se: a utilização de softwares para gestão da inovação e do conhecimento, o monitoramento de concorrentes e a qualificação e experiência do gerente. Com base na observação participante, não há tais softwares ou o monitoramento de concorrentes na empresa. Quanto à qualificação e experiência do gerente, Goslin e Naim (2009) apontam que os projetos ETO são geralmente complexos, o que leva a necessidade de um gerente com um nível de conhecimento elevado; o que não é fácil;

- Classificado pela maioria como neutro/inexistente os mecanismos de proteção, o controle financeiro, as estratégias de P\&D e de marketing, as características pessoais do diretor e a sua liderança transformacional. A falta de tolerância a falhas foi apontada por quatro respondentes como inexistente, ou seja, na visão deles existe sim uma tolerância a falhas. Isso pode ser explicado através do estudo de Rahim e Baksh (2003) no qual os autores chamam atenção para o fato da ETO ser caracterizada pelo volume de produção em pequenos lotes. Todo retrabalho gerado com as falhas no período da observação participante não tiveram nenhum impacto significativo na organização devido à baixa quantidade de peças/itens que precisaram ser refeitas. Tais retrabalhos puderam ser executados sem a necessidade de grandes investimentos;

- Unânime como inibidores têm-se: a falta de tempo por alta carga de trabalho, a política de treinamentos nula ou ineficiente e a falta de integração interfuncional. Os autores de ETO apontam para o tempo como um dos grandes problemas na ETO (PACAGNELLA; SILVA; PACÍFICO, 2014; WALTER; RIES, 1996; DONHA; RIBAS, 2016) e também ressaltam a importância da sobreposição de tarefas para essa tipologia (WALTER; RIES, 1996) que, para funcionar, precisa de uma forte integração interfuncional. Quanto aos treinamentos, estes são suprimidos devido ao departamento de projetos ETO trabalhar quase sempre no limite máximo de sua capacidade. Existe a ideia entre os 
funcionários de que a liderança enxerga os treinamentos como perda de tempo frente a grande carteira de trabalho;

- Classificados pela maioria como inibidores são: a ausência de gestão por resultados, o planejamento estratégico ineficiente, as resistências em contexto cultural, o senso de acomodação, a priorização de resultados de curto prazo, a rigidez de estruturas hierárquicas e a falta de um líder de projetos, os jogos políticos, os problemas de comunicação e a aversão exacerbada aos riscos. As constatações da observação participante apontam para alguns pontos relevantes: (1) nunca houve uma estratégia que cobrasse dos engenheiros/projetistas resultados sob 0 viés da inovação de produtos (a inovação só ocorria de acordo com o pedido de customização dos clientes); (2) a dificuldade do gerenciamento do ambiente multiprojetos que é caracterizado pela existência de projetos simultâneos (relacionados ou não) que se encontram em diferentes estágios, para clientes diferentes e com especificações diferentes (DANILOVIC; BÖRJESSON, 2001); (3) os mecanismos de proteção desenvolvido pelos funcionários que evitam correr riscos para manterem seus empregos, pois buscar a inovação incorre em riscos que as pessoas nem sempre estão dispostas a correr (CARAYANNIS; GONZALEZ, 2003; HADJIMANOLIS, 2003); (4) o tempo muito curto que não permite aos projetistas buscarem novas soluções e que leva o PDP à se tornar um processo de "manufatura de projetos"; (5) as tentativas mal sucedidas pela empresa em promover uma estrutura matricial devido às disputas entre a área funcional e o projeto (VALERIANO, 2005); (6) a dificuldade em elaborar um formulário de solicitação de projeto de customização devido à complexidade dos projetos ETO (GOSLIN; NAIM, 2009) e (7) o medo da liderança em arriscar com inovações que possam vir a tirá-los dos cargos que ocupam (CARAYANNIS; GONZALEZ, 2003).

O Quadro 4 sintetiza os resultados apresentados dividindo os fatores nas três classes previamente apresentadas (catalisadores à inovação de produtos no PDP ETO, inibidores à inovação de produtos no PDP ETO e os fatores que são neutros a inovação de produtos ou inexistentes no PDP ETO). 
Quadro 4 - Síntese dos resultados

(continua)

\begin{tabular}{|c|c|c|c|}
\hline $\begin{array}{c}\text { Fatores } \\
\text { catalisadores à } \\
\text { inovação de } \\
\text { produtos no PDP }\end{array}$ & $\begin{array}{c}\text { Fatores inibidores à } \\
\text { inovação de } \\
\text { produtos no PDP } \\
\text { ETO }\end{array}$ & $\begin{array}{r}\text { Fatores classificac } \\
\text { inovação de produtc } \\
\text { PDP }\end{array}$ & $\begin{array}{l}\text { os como neutros à } \\
\text { s ou inexistentes no } \\
\text { ETO }\end{array}$ \\
\hline $\begin{array}{l}\text { Desempenho passado } \\
\text { da empresa. }\end{array}$ & $\begin{array}{l}\text { Ausência de gestão } \\
\text { por resultados. }\end{array}$ & $\begin{array}{l}\text { Temor por canibalizar } \\
\text { a venda de produtos } \\
\text { já estabelecidos. }\end{array}$ & $\begin{array}{l}\text { Integração } \\
\text { interfuncional. }\end{array}$ \\
\hline $\begin{array}{l}\text { Estratégias de } \\
\text { diversificação. }\end{array}$ & $\begin{array}{l}\text { Planejamento } \\
\text { estratégico ineficiente. }\end{array}$ & $\begin{array}{l}\text { Mecanismos de } \\
\text { proteção. }\end{array}$ & $\begin{array}{l}\text { Centralização da } \\
\text { tomada de decisões } \\
\text { pelos líderes. }\end{array}$ \\
\hline $\begin{array}{l}\text { Existência de } \\
\text { exportações. }\end{array}$ & $\begin{array}{l}\text { Resistência em } \\
\text { contexto cultural. }\end{array}$ & $\begin{array}{l}\text { Sistema de } \\
\text { recompensas para } \\
\text { inovação com } \\
\text { incentivos P\&D. }\end{array}$ & $\begin{array}{l}\text { Fluxo de informações } \\
\text { eficiente. }\end{array}$ \\
\hline $\begin{array}{l}\text { Estratégia de } \\
\text { diferenciação. }\end{array}$ & $\begin{array}{l}\text { Senso de } \\
\text { acomodação. }\end{array}$ & $\begin{array}{l}\text { Existência de uma } \\
\text { estratégia de } \\
\text { orientação definida } \\
\text { que leve a um } \\
\text { planejamento claro. }\end{array}$ & Estratégias de P\&D. \\
\hline $\begin{array}{l}\text { Conjunto adequado } \\
\text { de pessoas. }\end{array}$ & $\begin{array}{l}\text { Falta de tempo por } \\
\text { alta carga de trabalho. }\end{array}$ & $\begin{array}{l}\text { Cultura e motivação } \\
\text { para a inovação. }\end{array}$ & $\begin{array}{l}\text { Estratégias de } \\
\text { marketing } \\
\text { (acompanhamento } \\
\text { das necessidades dos } \\
\text { clientes). }\end{array}$ \\
\hline Espírito de equipe. & $\begin{array}{l}\text { Priorização de } \\
\text { resultados de curto } \\
\text { prazo. }\end{array}$ & $\begin{array}{l}\text { Tempo suficiente para } \\
\text { se dedicar a inovação. }\end{array}$ & $\begin{array}{l}\text { Monitoramento de } \\
\text { concorrentes. }\end{array}$ \\
\hline $\begin{array}{l}\text { Aprendizado contínuo } \\
\text { e compartilhamento } \\
\text { de experiências. }\end{array}$ & $\begin{array}{l}\text { Ausência de uma } \\
\text { política de } \\
\text { treinamentos dos } \\
\text { recursos humanos. }\end{array}$ & $\begin{array}{l}\text { Existência de projetos } \\
\text { de longo prazo. }\end{array}$ & $\begin{array}{l}\text { Presença de um líder } \\
\text { de projetos. }\end{array}$ \\
\hline $\begin{array}{l}\text { Disponibilidade de } \\
\text { recursos financeiros. }\end{array}$ & $\begin{array}{l}\text { Rigidez de estruturas } \\
\text { hierárquicas. }\end{array}$ & Controle financeiro. & $\begin{array}{l}\text { Características } \\
\text { pessoais do diretor } \\
\text { executivo. }\end{array}$ \\
\hline $\begin{array}{l}\text { Existência de } \\
\text { indivíduos chave. }\end{array}$ & $\begin{array}{l}\text { Jogos políticos } \\
\text { internos e problemas } \\
\text { de obstrução por parte } \\
\text { de alguns } \\
\text { departamentos. }\end{array}$ & $\begin{array}{l}\text { Falta de recursos } \\
\text { financeiros. }\end{array}$ & $\begin{array}{l}\text { Diretor com liderança } \\
\text { transformacional. }\end{array}$ \\
\hline $\begin{array}{l}\text { Utilização de } \\
\text { equipamentos e } \\
\text { tecnologias } \\
\text { avançadas. }\end{array}$ & $\begin{array}{l}\text { Falta de integração } \\
\text { interfuncional. }\end{array}$ & $\begin{array}{l}\text { Falta de indivíduos } \\
\text { chave. }\end{array}$ & $\begin{array}{l}\text { Qualificação e } \\
\text { experiência do(s) } \\
\text { gerente(s). }\end{array}$ \\
\hline
\end{tabular}

Revista Produção Online. Florianópolis, SC, v.17, n. 4, p. 1379-1401, 2017. 
Quadro 4 - Síntese dos resultados

\begin{tabular}{|c|l|l|l|}
\hline $\begin{array}{c}\text { Fatores } \\
\text { catalisadores à } \\
\text { inovação de } \\
\text { produtos no PDP } \\
\text { ETO }\end{array}$ & $\begin{array}{c}\text { Fatores inibidores à } \\
\text { inovação de } \\
\text { produtos no PDP } \\
\text { ETO }\end{array}$ & \multicolumn{2}{|c|}{$\begin{array}{c}\text { Fatores classificados como neutros à } \\
\text { inovação de produtos ou inexistentes no } \\
\text { PDP ETO }\end{array}$} \\
\hline $\begin{array}{l}\text { Ausência de um líder } \\
\text { de projetos. }\end{array}$ & $\begin{array}{l}\text { Empoderamento dos } \\
\text { funcionários. }\end{array}$ & $\begin{array}{l}\text { Existência de uma } \\
\text { política de treinamento } \\
\text { dos recursos } \\
\text { humanos. }\end{array}$ & $\begin{array}{l}\text { Dirigentes com } \\
\text { habilidades que } \\
\text { identificam, apoiam, } \\
\text { incentivam e } \\
\text { assumem riscos em } \\
\text { prol da inovação. }\end{array}$ \\
\hline- & $\begin{array}{l}\text { Problemas na } \\
\text { comunicação entre } \\
\text { departamentos. }\end{array}$ & $\begin{array}{l}\text { Utilização de } \\
\text { softwares para gestão } \\
\text { da inovação e do } \\
\text { conhecimento. }\end{array}$ & $\begin{array}{l}\text { Falta de tolerância à } \\
\text { falha. }\end{array}$ \\
\hline- & $\begin{array}{l}\text { Falta de apoio e } \\
\text { aversão exacerbada } \\
\text { ao risco por parte dos } \\
\text { dirigentes. }\end{array}$ & $\begin{array}{l}\text { Promoção dos grupos } \\
\text { interfuncionais } \\
\text { orientado a projetos. }\end{array}$ & \\
\hline
\end{tabular}

\section{CONSIDERAÇÕES FINAIS}

$O$ estado atual da literatura contribui muito pouco para o entendimento do processo de inovação (BECHEIKH; LANDRY; AMARA, 2006). A literatura carece de estudos que integrem as pesquisas que buscam compreender o comportamento inovador nas organizações e que expliquem detalhadamente o desenvolvimento da capacidade de inovação de uma organização (VALLADARES; VASCONCELLOS; DI SERIO, 2014).

Diante do exposto é possível inferir que são escassos trabalhos que relacionem o conhecimento atual de fatores organizacionais que afetam à inovação com a capacidade de inovação de produtos no processo de desenvolvimento de um produto sob encomenda. Paralelamente, Grabenstetter e Usher (2015) afirmam que pouco existe sobre o ambiente de engenharia ETO na literatura. Isto precisa mudar, pois a tipologia de produção ETO está emergindo como uma das principais estruturas de cadeia de suprimentos e sua importância está aumentando à medida que a demanda por produtos customizados aumenta (GOSLIN; NAIM, 2009).

Assim, este trabalho ajuda a preencher esta lacuna ao passo que torna explícito os aspectos organizacionais que podem facilitar ou dificultar a inovação de produtos desenvolvidos sob encomenda. A partir deste estudo é possível extrair um conjunto de componentes que estão ligados ao sucesso e ao fracasso da inovação 
de produtos. Isso de acordo com a coleta de dados em uma empresa que utiliza a tipologia ETO e com o confronto destes dados com a literatura.

Cada vez mais se tornam importantes os estudos que envolvem a relação entre a prática da inovação e os fatores que a estimulam; já que a inovação tem sido considerada um tema estratégico e um fator crítico para a obtenção de vantagem competitiva (SANTOS et al., 2015). Além disso, tais estudos podem contribuir para que a alta administração tenha mais assertividade na promoção de comportamentos que estimulam a inovação. Lin e McDonough III (2011) ressaltam que este é um dever dos líderes.

Por isso, a principal contribuição deste trabalho é o fato de que ele deixa claros os principais aspectos que favorecem e os que não favorecem a inovação de produtos em uma empresa que trabalha sob a tipologia ETO. Isso pode ajudar administradores, gerentes e profissionais que trabalham com projetos de produtos ETO a fortalecer os fatores catalisadores e a combater os fatores inibidores à inovação.

Foram apontados como fatores internos à organização que favorecem a inovação, entre outros, a liberdade e a autonomia na condução de projetos, a disponibilidade de recursos financeiros, o aprendizado contínuo e o compartilhamento de experiências e a existência de indivíduos chave. Como inibidores foram identificados vários fatores, entre eles a falta de tempo por alta carga de trabalho, a política de treinamentos ineficiente e a falta de integração interfuncional. A classificação destes fatores em dois grupos distintos no PDP ETO é a principal contribuição deste trabalho.

Quanto às limitações da pesquisa, talvez a principal seja o fato da pesquisa se basear em um estudo de caso único. Portanto, como sugestão de trabalho futuro, seria interessante estender esta pesquisa para mais empresas que possuam um PDP ETO e, com isso, realizar comparações e propor generalizações.

\section{REFERÊNCIAS}

ALMEIDA, L. C. P.; ANDRADE, E. P.; ALENCAR R. S.; ASSIS, W. S.; SILVA A. M. Inovação em instituição militar de pesquisa: um estudo de caso exploratório. Revista Produção Online, Florianópolis, SC, v. 16, n. 4, p. 1371-1392, out./dez. 2016.

Http://doi.org/10.14488/1676-1901.v16i4.2353 
BAREGHEH, A.; ROWLEY, J.; SAMBROOK, S. Towards a multidisciplinary definition of innovation. Management Decision, v. 47, n. 8, p. 1323-1339, 2009.

https://doi.org/10.1108/00251740910984578

BECHEIKH, N.; LANDRY, R.; AMARA, N. Lessons from innovation empirical studies in the manufacturing sector: A systematic review of the literature from 1993-2003. Technovation, n.26, pp. 644-664, 2006.https://doi.org/10.1016/j.technovation.2005.06.016

BRUNO-FARIA, M. F.; FONSECA, M. V. A. Cultura de Inovação: Conceitos e Modelos Teóricos. RAC, Rio de Janeiro, v. 18, n.4, art.1, pp. 372-396, Jul./Ago. 2014. http://dx.doi.org/10.1590/1982-7849rac20141025

CARAYANNIS, E. G.; GONZALEZ. E. Creativity and innovation = competitiveness? When, how and why. In: SHAVININA, L.V. (Org.), The International Handbook on Innovation. Oxford: Elsevier Science. Parte VIII, cap. 3, p. 587- 606, 2003. https://doi.org/10.1016/B978-008044198-6/50040-1

CHRYSSOCHOIDIS, G. Factors affecting product innovations: A literature review. Agricultural Economics Review, v.4, n. 1, p. 47-62, Jan. 2003.

CROSSAN, M. M.; APAYDIN, M. A Multi-Dimensional Framework of Organizational Innovation : A Systematic Review of the Literature. Journal of Management Studies, September, v. 47, n. 6, pp. 1154-1191, 2010. https://dx.doi.org/10.1111/j.1467$\underline{6486.2009 .00880 . x}$

D’ESTE, P.; IAMMARINO, S.; SAVONA, M.; TUNZELMANN, N. V. What hampers innovation? Revealed barriers versus deterring barriers. Research Policy, n.41, n. 2, p. 482-488, 2012. https://doi.org/10.1016/j.respol.2011.09.008

DAMANPOUR, F.; WISCHNEVSKY, D. Research on innovation in organizations: Distinguishing innovation generating from innovation-adopting organizations. Journal of Engineering and Technology Management JET-M. , United States, v.23, p.269-291, 2006. https://doi.org/10.1016/j.jengtecman.2006.08.002

DANILOVIC, M.; BÖRJESSON, H. Managing the Multiproject Environment. In: The Third Dependence Structure Matriz International Workshop, Massachusetts: Institute of Technology (MIT), 2001.

DEMIRBAS, D.; HUSSAIN, J.G.; MATLAY, H. Owner-managers' perceptions of barriers to innovation: empirical evidence from Turkish SMEs. Journal of Small Business and Enterprise Development, v. 18, n. 4, p. 764 - 780, 2011. https://doi.org/10.1108/14626001111179794

DEMO, P. Metodologia científica em ciências sociais. 3. ed. São Paulo: Atlas, 1995.

DONHA, R. G.; RIBAS, M. A. S. Proposta de Melhoria no Gerenciamento de Tempo do Projeto de Produtos Desenvolvidos sob a Tipologia de Produção Engineer to Order a partir do PMBOK®: um estudo de caso. ENCONTRO NACIONAL DE ENGENHARIA DE PRODUÇÃO, 36.,2016. Anais .... João Pessoa: Abepro, 2016.

FLYNN, B., SAKAKIBARA,S., SCHROEDER, R. G., BATES,K. A., FLYNN, E. J. Empirical research methods in operations management. Journal of Operations Management, v. 9, n. 2, p. 250-284, 1990. https://doi.org/10.1016/0272-6963(90)90098-X 
FOGLIATTO, F.S.; SILVEIRA, G.J.C.; BORENSTEIN, D. The mass customization decade: an update review of the literature. International Journal Production Economics, 138, 142, 2012. https://doi.org/10.1016/j.ijpe.2012.03.002

GIL, A. C. Como elaborar projetos de pesquisa. 4. ed. São Paulo: Atlas, 2002.

GOSLING, J., NAIM, M.M. Engineer-to-order supply chain management: a literature review and research agenda. International Journal of Production Economics, v. 122, n. 2, p. 741-754, 2009. https://doi.org/10.1016/.i.ipe.2009.07.002

GRABENSTETTER, D. H.; USHER, J. M. Sequencing jobs in an engineer-to-order engineering environment. Production \& Manufacturing Research, v.3, n.1, p.201-217, 2015. http://dx.doi.org/10.1080/21693277.2015.1035461

GUNDAY, G.; ULUSOY, G.; KILIC, K.; ALPKAN, L. Effects of innovation types on firm performance. International Journal of Production Economics, v.133, p. 662-676, 2011. https://doi.org/10.1016/j.ijpe.2011.05.014

HADJIMANOLIS, A. The barriers approach to innovation. In: SHAVININA, L.V. (Org.), The International Handbook on Innovation. Oxford: Elsevier Science. Parte VIII, cap. 1, p. 559573, 2003. https://doi.org/10.1016/B978-008044198-6/50038-3

HAGE, J.; MEEUS, M. Innovation, science, and institutional change: a research handbook. New York: Oxford University Press, 2009.

HAIR JUNIOR, J. F;, ANDERSON, R. E.; TATHAM, R. L.; BLACK, W. C. Análise multivariada de dados. 6 ed. Porto Alegre: Bookman, 2009. 688 p.

HAUSER, J.; TELLIS, G. J.; GRIFFIN, A. Research on Inovation: A Review and Agenda for Marketing Science. Marketing Science, v. 25, n. 6, p. 687-717, 2006.

https://doi.org/10.1287/mksc.1050.0144

HOOSHMAND, Y.; KÖHLER, P.; KORFF-KRUMM, A. Cost Estimation in ETO Manufacturing. Open Engineering, v.6, n.1, p. 22-34, 2016.

https://doi.org/10.1515/eng-2016-0002

JANTZ, R.C. The Determinants of Organizational Innovation: An Interpretation and Implications for Research Libraries. College \& Research Libraries, pp.crl14-580, 2014. https://doi.org/10.5860/crl.76.4.512

LAM, A. Organizational innovation. Brunel Research in Enterprise, Innovation, Sustainability, and Ethics. Londres: Brunel University. Apr. 2004. https://doi.org/10.1093/oxfordhb/9780199286805.003.0005

LEONTITSIS, A.; PAGGE, J. A simulation approach on Cronbach's alpha statistical significance. Mathematics and Computers in Simulation. v. 73, p. 336-340. 2007. https://doi.org/10.1016/j.matcom.2006.08.001

LIN, H.; McDONOUGH III, E. F. Investigating the role of leadership and organizational culture in fostering innovation ambidexterity. IEEE Transactions on engineering management, v.58, n.3, p.497-507, 2011. https://doi.org/10.1109/TEM.2010.2092781 
MACHADO, D. D. P. N.; VASCONCELLOS, M. A. Organizações Inovadoras: Existe Uma Cultura Específica Que Faz Parte Deste Ambiente? Revista de Gestão USP, São Paulo, v.14, n.4, p.15-31, 2007.

MADRID-GUIJARRO, A.; GARCIA, D. VAN AUKEN, H. Barriers to Innovation among Spanish manufacturing SMEs. Journal of Small Business Management, v. 47, n.4, p. 465488, 2009. http://doi.org/10.1111/j.1540-627X.2009.00279.x

MARZALL, L. F.; SANTOS L. A.; GODOY L. P. Inovação no projeto de produto como fator para redução de custos logísticos e de produção. Revista Produção Online, Florianópolis, SC, v. 16, n. 1, p. 342-365, jan./mar. 2016. http://doi.org/10.14488/1676-1901.v16i1.2168

MAZZOTTI A.J.A., GEWANDSZNAJDER F. 0 método nas ciências naturais e sociais. São Paulo: Pioneiras; 1998.

MELO, J. C. F.; BAGNO, R. B. Atribuições de um time dedicado à gestão da inovação em um grupo industrial brasileiro: um estudo longitudinal baseado em eventos. Revista

Produção Online. Florianópolis, SC, v.17, n. 2, p. 482-506, 2017.

https://doi.org/10.14488/1676-1901.v17i2.2376

MORGAN, G. Imagens da organização. São Paulo: Atlas, 2002.

OECD/Eurostat. Proposed guidelines for collecting and interpreting technological innovation data: Oslo Manual. Paris, 2005.

PACAGNELLA JUNIOR, A. C.; SILVA, S. L.; PACIFICO, O. . Fatores Críticos de Sucesso em Projetos de Bens de Capital com Tipologia Engineering-to-Order: Uma análise a partir da literatura. Iberoamerican Journal of Project Management (IJoPM), v. 5, p. 01, 2014.

PISTORIUS, C. W. I.; UTTERBACK, J. M. Multi-mode interaction among technologies. Research Policy, v.26, p.67-84, 1997. https://doi.org/10.1016/S0048-7333(96)00916-X

RAHIM, A. R. A.; BAKSH, M. S. N. The need for a new product development framework for engineer-to-order products. European Journal of Innovation Management, v.6, n.3, p.182196, 2003. https://doi.org/10.1108/14601060310486253

ROZENFELD, H.; FORCELLINI, F. A.; AMARAL, D. C.; TOLEDO, J. C.; SILVA, S. L.; ALLIPRANDINI, D. H.; SCALICE, R. K. Gestão de desenvolvimento de produtos: uma referência para a melhoria do processo. São Paulo: Saraiva, 2006.

SANTOS, G. E. T.; GUIMARÃES, M. R. N.; FONTES, A. R. M.; SALTORATO, P.; DE ANDRADE, L. C. M. Relação entre cultura organizacional e inovação tecnológica - um estudo de caso em uma fábrica de fitas adesivas. Revista GEINTEC, v. 5, n. 2, p. 2000 2012, 2015. https://doi.org/10.7198/S2237-0722201500020006

SODHI, M. S.; TANG, C. S. Guiding the next generation of doctoral students in operations management. International Journal of Production Economics, v. 150, p. 28-36, 2014. https://doi.org/10.1016/j.ijpe.2013.11.016

STEFANOVITZ, J. O.; NAGANO, M. S. Gestão da inovação de produto: proposição de um modelo integrado. Production, v. 24, n. 2, p. 462-476, abr./jun. 2014.

http://dx.doi.org/10.1590/S0103-65132013005000062 
TIDD, J.; BESSANT, J.; PAVITT, K. Gestão da inovação. 3. ed. Porto Alegre: Bookman, 2008.

VALENCIA, J. C. N.; VALLE, R. S.; JIMÉNEZ, D. J.; Organizational culture as determinant of product innovation. European Journal of Innovation Management, v.13, n. 4, p. 466-480, 2010. https://doi.org/10.1108/14601061011086294

VALERIANO, D. Moderno gerenciamento de projetos. 2. ed. São Paulo: Prentice Hall, 2015.

VALLADARES, P.S.D.; VASCONCELLOS, M.A.; DI SERIO, L.C. Capacidade de inovação: revisão sistemática da literatura. RAC - Revista de Administração Contemporânea, vol. 18, n. 5, set./out., pp. 598-626, 2014.http://dx.doi.org/10.1590/1982-7849rac20141210

WALTER, C.; RIES, O. A Automação da engenharia de produto em um ambiente ETO/OKP. Máquinas e Metais. Ago., p.132-139, 1996.

WANG, T.; ZHAO, B.; THORNHILL, S. Pay dispersion and organizational innovation: The mediation effects of employee participation and voluntary turnover. Human Relations, v. 68, n. 7, p. 1155-1181, 2015. https://doi.org/10.1177/0018726715575359

WILLNER, O.; POWELL, D.; GERSCHBERGER, M.; SCHÖNSLEBEN, P. Exploring the archetypes of engineer-to-order: an empirical analysis. International Journal of Operations \& Production Management, v. 36, n.3, pp. $242-264,2016$.

https://doi.org/10.1108/IJOPM-07-2014-0339

YIN, R. K. Estudo de caso: planejamento e métodos. 4 ed. Porto Alegre: Bookman, 2010.

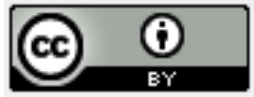

Artigo recebido em 03/01/2017 e aceito para publicação em 30/10/2017

DOI: http://dx.doi.org/10.14488/1676-1901.v17i4.2678 